\title{
NOTES ON THE RATIONAL PLANE CUBIC CURVE*
}

\author{
BY T. W. MOORE
}

1. Involutions. The rational cubic curve in the plane can be defined parametrically by three equations of the type

$$
x_{i}=\left(\alpha_{i} t\right)^{3}, \quad(i=1,2,3) .
$$

Then any line $(\xi x)=0$ will determine the binary cubic

$$
(a \xi)(\alpha t)^{3}=0,
$$

on the curve and we have an involution of line sections. There is a unique binary cubic $(\beta t)^{3}=0$, called the fundamental cubic of the $R_{2}^{3}$, apolar to each of the three cubics (1) and hence apolar to any line section. The condition that any three points of the base cubic $R_{2}^{3}$ be on a line is that their parameters satisfy the relation

$$
\beta_{0} s_{3}+\beta_{1} s_{2}+\beta_{2} s_{1}+\beta_{3}=0,
$$

the polarized form of $(\beta t)^{3}=3$. The roots of $(\beta t)^{3}=0$ are the triple points of the involution of line sections and are thus the parameters of the flexes.

If we denote by $(\gamma t)^{3}$ the cubic covariant of $(\beta t)^{3}$, then the polarized form of $(\gamma t)^{3}=0$ represents another cubic involution $I_{2,1}^{2}$, on the base curve, of which the roots of the cubic covariant are triple points. These three points are the sextactic points, contacts of the tangents from the flexes, and consequently the intersections of the harmonic polars with the curve. Winger $\dagger$ has shown that this involution is determined by the contacts of the tri-tangent conics. The tri-tangent conics are the envelopes of lines joining points apolar to given fixed pairs of points of the base cubic. But what is more important here is the fact that this involution of points is also cut out of the cubic by the net of conics on the three sextactic points. We can see

* Presented to the Society, May 1, 1926.

† R. M. Winger, this Bulletin, vol. 25 (1918-19), pp. 27-34. 
this from the condition that $3 n$ points of the curve $R_{2}^{3}$ be on an $n$-ic, and here we find that the three sextactic points and the three points of contact of any tri-tangent conic are on a conic. Therefore we have the following theorem.

THEOREM. The involution of points apolar to the cubic covariant of the fundamental cubic is determined by the net of conics on the sextactic points.

It may be noted that the net of conics on any three points of the cubic $R_{2}^{3}$ cut out a cubic involution.

2. Inversion of the Curve. Let us give the flex points the parameters $0,1, \infty$, thus determining the sextactic points at $t=2$, $-1, \frac{1}{2}$. If we write the equation of the curve referred to the sextactic points as reference points, we find its equation to be

$$
\sum x_{i}^{2} x_{j}-6 x_{1} x_{2} x_{3}=0, \quad i, j=1,2,3, \quad i \neq j,
$$

or

$$
\left(x_{1}+x_{2}\right)\left(x_{2}+x_{3}\right)\left(x_{3}+x_{1}\right)-8 x_{1} x_{2} x_{3}=0 ;
$$

and its Hessian is

$$
8 \sum_{i=1}^{3} x_{i}^{8}-9 \sum^{6} x_{i}^{2} x_{j}+30 x_{1} x_{2} x_{3}=0 .
$$

From the form of equation (4) it is evident that the equation is unchanged by the quadratic transformation $x_{i} y_{i}=1, i=1,2,3$. Hence we have the following theorem.

THEOREM. The base cubic, when referred to the sextactic points as a reference triangle, is inverted into itself by the quadratic transformation $x_{i} y_{i}=1, i=1,2,3$.

This transformation interchanges the lines of the plane and the net of conics on the vertices of our reference triangle, or in other words, the two cubic involutions determined by the line sections and the conics on the sextactic points are interchanged. In this scheme of reference the unit point and unit line are the double point and line of flexes of the cubic respectively. In the inversion the line of flexes is sent into the conic $N$, the involution conic of the node, and conversely. The tri-tangent conics touching the base cubic in three points are transformed into rational quartic curves which have double points on the 
vertices of the reference triangle and which have contact with the cubic at three other collinear points.

3. A Pencil of Cubics. There is a noteworthy configuration connected with the cubic (4) and its Hessian. Let us consider the pencil of cubics

(6) $\lambda\left(\sum^{6} x_{i}^{2} x_{j}-6 x_{1} x_{2} x_{3}\right)-2 \mu\left(\sum_{1}^{3} x_{i}^{3}-\sum^{6} x_{i}^{2} x_{j}+3 x_{1} x_{2} x_{3}\right)=0$, of which the Hessian is a member. The cubic (4) has its double point at $(1,1,1)$ and the triangle with its vertices at the sextactic points is known to be perspective with the triangle of flex tangents, the axis of perspection being the line of flexes, the unit line. The triangle of sextactic points is in fact perspective in four ways with the triangle of flex tangents and will have in consequence all the properties of such triangles. The lines joining corresponding vertices in the perspection in question are the harmonic polars $x_{2}-x_{3}=0, x_{3}-x_{1}=0, x_{1}-x_{2}=0$, and the center of perspection is the unit point.

Now every member of the pencil (6) has the same double point and the same flex points and line of flexes. Every member of the pencil will meet the harmonic polars in the points $(\lambda, \mu, \mu),(\mu, \lambda, \mu),(\mu, \mu, \lambda)$ and will have its triangle of sextactic points perspective with its triangle of flex tangents. Conversely, for any triangle of this system of perspective triangles, there is one curve of the pencil whose flex tangents are the sides of the triangle and another curve of the same pencil for which the harmonic polars meet the curve at the vertices of this triangle. Hence there is a perspective collineation with the double point $(1,1,1)$ as a point of fixed lines and the line of flexes $x_{1}+x_{2}+x_{3}=0$ as a line of fixed points transforming the triangle of sextactic points of the base cubic (4) into the triangle having vertices at $(\lambda, \mu, \mu),(\mu, \lambda, \mu),(\mu, \mu, \lambda)$.

4. Osculants of the Cubic. For convenience we shall now give to the flexes the parameters $1, \omega, \omega^{2}$, thus giving the double point the parameters $0, \infty$, and we shall take the line of flexes and the tangents at the double point as a reference triangle. Then the equations of the curve $R_{2}^{3}$ may be written 


$$
x_{1}=t^{3}-1, \quad x_{2}=-3 t^{2}, \quad x_{3}=3 t .
$$

The map equation of the cubic is then

$$
\xi_{1} t^{3}-3 \xi_{2} t^{2}+3 \xi_{3} t-\xi_{1}=0
$$

and the condition for three points of the cubic to form a line section is

$$
S_{3}=1 \text {. }
$$

The first osculant of (7) at $t=t_{1}$ is, by definition,

$$
x_{1}=t_{1} t^{2}-1, \quad x_{2}=-\left(t^{2}+2 t t_{1}\right), \quad x_{3}=2 t+t_{1} .
$$

From Thomsen's* consideration of the dual case we know that the Hessian $H$ of (8) is the line equation of the osculant conic (10). If we polarize the Hessian $H$, giving

$$
\left(\xi_{1} \xi_{3}-\xi_{2}^{2}\right) t_{1} t_{2}+\left(\xi_{2} \xi_{3}-\xi_{1}^{2}\right) \frac{t_{1}+t_{2}}{2}+\left(\xi_{1} \xi_{2}-\xi_{3}^{2}\right)=0,
$$

we get the net of conics on the three flex tangents. For given $t_{1}$ and $t_{2}$ the particular conic has contact with the tangents to $R_{2}^{3}$ at $t_{1}$ and $t_{2}$. From (10) we can get the second osculant of (7) with respect to $t_{1}$ and $t_{2}$ as

$$
x_{1}=t_{1} t_{2} t-1, \quad x_{2}=-\left(t_{1}+t_{2}\right) t-t_{1} t_{2}, \quad x_{3}=t+t_{1}+t_{2} .
$$

This is called the mixed linear osculant with respect to $t_{1}$ and $t_{2}$ and we see that this line meets the line $t_{1} t_{2}$ on the line of flexes. Morley $\dagger$ has shown that the three mixed linear osculants of $t_{2}, t_{3} ; t_{3}, t_{1} ; t_{1}, t_{2}$ meet in a point, and thus associated with any three points on the curve is some point in the plane, whose coordinates are $x_{1}=s_{3}-1, x_{2}=-s_{2}, x_{3}=s_{1}$, where the $s^{\prime}$ s are symmetric functions of the three $t$ 's. On the other hand, given a point $(x)$ in the plane, the points $t_{1}, t_{2}, t_{3}$ on the curve are given as roots of the cubic equation

$$
t^{3}-x_{3} t^{2}-x_{2} t-\left(x_{1}+1\right)=0 \text {. }
$$

* Thomsen, Osculants of plane rational quartic curves, AMERICAN JOURNAL, vol. 32, p. 228.

$\dagger$ F. Morley, On reflexive geometry, Transactions of this Society, vol. 8, p. 16. 
A given line ( $\xi$ ) determines on $R_{2}^{3}$ the binary cubic (8) whose Hessian points are given by $H=0$. To find the Hessian points for a given $(\xi)$, consider the pencil of conics on the flex tangents and the line $(\xi)$. This pencil sets up an involution of pairs of common lines with the base chive. Hence two conics of the pencil will have contact with $R_{2}^{3}$ at $t_{1}$ and $t_{2}$, one at each of the required points.

Conversely, a binary quadratic on the curve, represented by points $t_{1}$ and $t_{2}$, is in general apolar to but one line section, or represents the Hessian points of this line section. This line is the remaining common tangent of the osculant conics at $t_{1}$ and $t_{2}$ and is the mixed linear osculant of $t_{1}$ and $t_{2}$. The exceptions to the rule occur when we consider the syzygetic pencils of binary cubics formed by the pencils of lines on the vertices of the triangle of flex tangents. Here both the cubic and its cubic covariant are line sections and every member of this pencil has the same Hessian.

The osculant conic at $t_{1}$ meets the cubic in four more points which form a self-apolar set, ${ }^{*}$ and whose parameters are given here by

$$
t_{1}^{2} t-4 t^{3}+6 t_{1} t^{2}-4 t_{1}^{2} t+1=0 .
$$

We observe that the first polars of this quartic are represented by line sections on the point $\left(0,-t_{1}, 1\right)$. Hence we have the following theorem.

THEOREM. The self-apolar quartic, given by the remaining intersections of the $R_{2}^{3}$ and its osculant conic at $t_{1}$, has its pencil of first polars represented by the pencil of line sections containing the line of flexes and the line joining the point $t_{1}$ to the double point.

5. Osculants of the Line Form of the Cubic. Let us investigate the line form of equation (7). The equations are

$$
\xi_{1}=3 t^{2}, \quad \xi_{2}=2 t^{3}+1, \quad \xi_{3}=t\left(t^{3}+2\right) .
$$

The first osculant of (15), in line form, is (16) $\xi_{1}=3\left(t^{2}+t_{1} t\right), \quad \xi_{2}=t^{3}+3 t_{1} t^{2}+2, \quad \xi_{3}=2 t_{1} t^{3}+3 t+t_{1}$.

* Thomsen, loc. cit., p. 213. 
This is a rational line cubic or point quartic curve, and is the parametric form of the cubic covariant $J$ of (8).*

The polarized form of $J$ gives the system of all line cubics whose members have contact with $R_{2}$ at the points of inflection, and for a given $t_{1}, t_{2}, t_{3}$, the curve has contact with the tangent lines to $R_{2}^{3}$ at $t_{1}, t_{2}, t_{3}$ respectively. The system is given by the equation

$$
\begin{gathered}
\left(-\xi_{1}^{3}+3 \xi_{1} \xi_{2} \xi_{3}-2 \xi_{2}^{3}\right) s_{3}+\left(\xi_{1}^{2} \xi_{2}+\xi_{2}^{2} \xi_{3}-2 \xi_{3}^{2} \xi_{1}\right) s_{2} \\
+\left(\xi_{2} \xi_{3}^{2}+\xi_{3} \xi_{1}^{2}-2 \xi_{1} \xi_{2}^{2}\right) s_{1}+\left(-\xi_{1}^{3}+3 \xi_{1} \xi_{2} \xi_{3}-2 \xi_{3}^{3}\right)=0 .
\end{gathered}
$$

The point form of (16) is

$$
\begin{aligned}
& x_{1}=-2\left(t_{1}^{2} t^{4}-t^{3}-t_{1}^{2} t+1\right), \\
& x_{2}=2 t_{1} t^{4}+4 t_{1}^{2} t^{3}-3 t^{2}-2 t_{1} t-t_{1}^{2}, \\
& x_{3}=-\left(t^{4}+2 t_{1} t^{3}+3 t_{1}^{2} t_{2}-4 t-2 t_{1}\right),
\end{aligned}
$$

which is a rational quartic curve. The tangent to $R_{2}^{3}$ at $t_{1}$ is a double line of this quartic, having its second contact with the quartic on the line of flexes. It is interesting to note that the tangent at $t_{1}$ meets the base curve again at the point whose parameter is $1 / t_{1}^{2}$ and that the second contact of the osculant curve with this line has the same parameter value.

This osculant degenerates when $t_{1}$ is a sextactic point. Thus, if $t_{1}=-1$, the equations (16) and (18) become

$$
\text { (19) }\left\{\begin{array} { l } 
{ \xi _ { 1 } = 3 t ( t - 1 ) , } \\
{ \xi _ { 2 } = ( t ^ { 2 } - 2 t - 2 ) ( t - 1 ) , } \\
{ \xi _ { 3 } = - ( 2 t ^ { 2 } + 2 t - 1 ) ( t - 1 ) ; }
\end{array} \text { (20) } \left\{\begin{array}{l}
x_{1}=2\left(t^{2}+t+1\right)(t-1)^{2}, \\
x_{2}=\left(2 t^{2}+1\right)(t-1)^{2}, \\
x_{3}=\left(t^{2}+2\right)(t-1)^{2} .
\end{array}\right.\right.
$$

Thus the corresponding point of inflection $t=1$ and the square of the flex tangent respectively have factored out. Furthermore this conic is a tri-tangent conic, for it has contact with $R_{2}^{3}$ at the sextactic point and at the remaining flexes $t=\omega, \omega^{2}$.

YALE UNIVERSITY

* Thomsen, loc. cit., p. 228. 